๑ Open Access Full Text Article

ORIGINAL RESEARCH

\title{
Suppressing UVRAG Induces Radiosensitivity by Triggering Lysosomal Membrane Permeabilization in Hypopharyngeal Squamous Cell Carcinoma
}

This article was published in the following Dove Press journal:

OncoTargets and Therapy

\section{Jianwen Wang \\ Xuehai Wang \\ Kai Liu \\ Li Gu \\ Lei Yu \\ Li Han \\ Zhaojin Meng}

Department of Otolaryngology, Weihai Municipal Hospital, Cheeloo College of Medicine, Shandong University, Weihai 264200, Shandong, People's Republic of China
Correspondence: Xuehai Wang Department of Otolaryngology, Weihai Municipal Hospital, Cheeloo College of Medicine, Shandong University, Weihai 264200, Shandong, People's Republic of China

Email xuehai58I@I63.com
Introduction: Radiotherapy is one of the most important methods in the treatment of patients with hypopharyngeal squamous cell carcinoma (HSCC). However, radioresistance will be developed after repeated irradiation. Among many key factors contributing to radioresistance, enhanced autophagy is recognized as one of the most important. The ultraviolent irradiation resistance-associated gene (UVRAG) is reported to be a crucial gene involved in the process of autophagy. Here, we test whether UVRAG has effect on the radioresistance of HSCC.

Methods: HSCC cell line Fadu cells were treated with irradiation to test levels of autophagy. Tumor tissues from primary and recurrent HSCC patients were tested by immunohistochemistry. Then, we knocked down UVRAG to test its role in cell growth and the malignant behaviors. Response of cells to treatment was examined using LDH release assay, immunofluorescence, Western blot analysis and colony formation.

Results: We found that irradiation induced autophagy in Fadu cells. Immunohistochemistry of primary and irradiated HSCC tumor tissues showed that UVRAG was upregulated after irradiation treatment. Inhibiting UVRAG with siRNA interfered cell growth, cell cycle, malignant behaviors and autophagic flux in Fadu cells. Knocking down UVRAG increased DNA damage and cell death induced by irradiation. Finally, we found that inhibiting UVRAG induced lysosomal membrane permeabilization, which contributed to radiosensitization of Fadu cells.

Conclusion: Our findings supported the oncogenic properties of UVRAG in HSCC and inhibiting UVRAG increased radiosensitivity in HSCC by triggering lysosomal membrane permeabilization. Therefore, UVRAG might be a promising target in the treatment of HSCC.

Keywords: UVRAG, autophagy, radiosensitivity, hypopharyngeal squamous cell carcinoma, lysosomal membrane permeabilization

\section{Introduction}

Hypopharyngeal squamous cell carcinoma (HSCC) accounts for about 5\% of head and neck tumors, and symptoms of HSCC patients are non-specific. ${ }^{1}$ As a result, many HSCC patients are usually diagnosed in advanced stages. ${ }^{2}$ According to global cancer statistics 2018, 80,608 new cases of HSCC were diagnosed and 34,984 patients of HSCC died in $2018 .^{3}$ Radiotherapy is recognized as one of the most important methods in the treatment of HSCC. ${ }^{4}$ But data show that the 5 -year survival rate for HSCC patients who have only received radiotherapy is less than 
$20 \%$. Radioresistance is one of the main factors contributing to the treatment failure of HSCC patients. ${ }^{4}$ Although many studies have been done to find out the factors leading to radioresistance of various tumor types, the underlying mechanisms of the radioresistance of HSCC are still unclear.

Radiotherapy has been used in the clinic for more than one century. Its anti-tumor efficiency in many malignant neoplasms has been well approved. But radioresistance always occurs after repeated treatment. Until now, many factors have been recognized leading to radioresistance, such as cell cycle arrest, apoptotic resistance, enhanced DNA damage repair and autophagy. ${ }^{5,6}$ A recent study by Zhou et al found that lysosomal membrane permeabilization (LMP), a lysosome-dependent cell death, is also involved in the radiosensitization of cancer. ${ }^{7}$ In the process of LMP, lysosomal enzymes are released into the cytoplasm through the impaired lysosomal membrane and programmed cell death is induced. ${ }^{7}$

Autophagy is a lysosome-dependent process. $^{8}$ An increasing number of studies showed that autophagy is a protective process for tumor cells. ${ }^{9-11}$ Its role in response to radiotherapy has been reported by various researchers. Many studies found that radiotherapy enhanced autophagic flux and combining autophagy inhibitors increased radiosensitivity in cancers. ${ }^{12-14}$

Ultraviolet irradiation resistance-associated gene (UVRAG), a mammalian homolog of yeast Vps38 with oncogenic properties and located on the 11q13 chromosome band, a relevant area with oncogenic implications in many cancers, is an important factor involving in autophagy regulation. ${ }^{15-17}$ It has the function of promoting autophagosome formation and maturation by targeting the Beclin 1/PtdIns3KC3 (class III phosphatidylinositol3-kinase) complex. ${ }^{18}$ UVRAG is also reported to be involved in regulating the function and morphology of lysosomes. ${ }^{19}$ A recent study found that UVRAG is involved in DNA damage repair. ${ }^{20}$ Inhibiting UVRAG increased radiosensitivity in pancreatic cancer and colorectal cancer cells. ${ }^{21,22}$ But the underlying mechanisms are not well illustrated.

Whether UVRAG has the same effect on HSCC is largely unknown. In this study, we hypothesized that UVRAG plays a role in the promotion of cell growth and radioresistance of HSCC cell line Fadu cells. We tried to figure out the role of UVRAG in autophagy, cell growth, malignant behaviors and radioresistance of HSCC. HSCC tumor tissues from primary HSCC patients and recurrent HSCC patients who have only received radiotherapy were also examined.

\section{Materials and Methods Cell Culture}

Hypopharyngeal squamous cell carcinoma cell line Fadu cells were purchased from the Culture Collection of the Chinese Academy of Sciences (Shanghai, China) and cultured in DMEM (ThermoFisher Scientific; Waltham, MA, USA) supplemented with $10 \%$ fetal bovine serum (FBS; ThermoFisher Scientific).

\section{Western Blot Analysis}

Fadu cells were treated with or without 4 Gy irradiation at a dose rate of $1.8 \mathrm{~Gy} / \mathrm{min}$ in a linear accelerator (Primus Hi, Siemens Medical Instruments; Berlin, Germany). Twenty-four-hour later, cells were lysed in RIPA buffer (ThermoFisher Scientific) and lysates $(20 \mu \mathrm{g})$ were analyzed on SDS-PAGE. Western blot analysis was performed as previously described. ${ }^{8}$ The following antibodies were used: LC3B, P62, GAPDH, UVRAG (Cell Signaling Technology; Danvers, MA, USA).

\section{GFP-LC3 Transient Transfection}

The pSELECT-GFP-LC3 plasmid (Genepharma; Shanghai, China) was used to study the formation of autophagosomes. Briefly, Fadu cells were transfected with the plasmid by using Lipofectamine 2000 reagent (ThermoFisher Scientific). After being treated with or without 4 Gy irradiation, GFP-LC3 puncta per cell was counted under a Leica TCS SP5 Confocal Laser Scanning Microscope (Leica Microsystems, Wetzlar, Germany).

\section{LysoTracker Staining}

LysoTracker Red (66 nM) was added to Fadu cells pretreated with or without 4 Gy irradiation. Nuclei were stained with DAPI (ThermoFisher Scientific). Fluorescence was observed under confocal microscopy with a Leica SP5 Confocal Microscope.

\section{Cathepsin B Activity Assay}

Activity of cathepsin B was tested with a cathepsin B activity assay kit (Abcam, Cambridge, UK). Briefly, Fadu cells treated with 4 Gy irradiation or control cells were lysed, and supernatants were incubated with cathepsin B substrates. Fluorescence was measured on a microplate 
reader (PerkinElmer; San Jose, CA, USA) at excitation/ emission wavelengths $=400 / 505 \mathrm{~nm}$.

\section{Immunohistochemistry}

Three matched HSCC tumor tissues from primary HSCC patients and recurrent HSCC patients who have only received radiotherapy were collected in the Department of Otolaryngology, Weihai Municipal Hospital. The use of human tissue samples was approved by the Research Ethics Committee of Shandong University and performed according to relevant guidelines and regulations. All patients provided informed consent, in accordance with the Declaration of Helsinki.

Paraffin-embedded hypopharyngeal squamous cell carcinoma samples were sectioned $(4 \mu \mathrm{m})$ and mounted onto microscopic slides. Deparaffinized sections were incubated with the primary antibody at $4^{\circ} \mathrm{C}$ overnight (UVRAG, Cell Signaling Technology), rinsed with PBS, and incubated with goat anti-rabbit secondary antibody (ZSGB-Bio; Beijing, China). Images were taken by a Leica DMi8 microscope (Leica Microsystems). Results of immunohistochemistry were quantified as follows: 0 , no staining; 1 , weak staining in $<50 \%$ cells; 2 , weak staining in $\geq 50 \%$ cells; 3 , strong staining in $<50 \%$ cells; and 4 , strong staining in $\geq 50 \%$ cells.

\section{Transmission Electron Microscopy}

Transmission electron microscopy was used to examine the ultrastructure of Fadu cells treated with or without 4 Gy irradiation. Experiments were carried out as described previously. ${ }^{8}$ Cells were observed with a JEM1200EX II electron microscope (JEOL; Tokyo, Japan).

\section{Transfection of siRNA}

Fadu cells were transfected with $20 \mathrm{nM}$ siRNA by using lipofectamine 2000 according to the manufacturer's protocol. Sequences for the siRNAs were the following: negative control, 5'- UUCUCCGAACGUGUCACGU TT-3'; UVRAG, 5'- UCACUUGUGUAGUACUGAA-3' (Genepharma, Shanghai, China). Knocking down efficiency was analyzed by Western blot.

\section{Cell Viability}

Fadu cells transfected with negative control or UVRAG siRNA were plated into 96-well plates and incubated overnight. Cell viability was assessed with the CCK-8 assay.

\section{EdU Assay}

Fadu cells transfected with negative control or UVRAG siRNA were seeded into 24-well, flat-bottomed plates. Cell proliferation was assessed with the EdU Assay Kit (Ribobio; Guangzhou, China). Images were acquired under a Leica DMi8 fluorescence microscopy.

\section{Cell Migration and Invasion Assays}

Cell migration of Fadu cells treated with control siRNA or UVRAG siRNA was tested by wound healing assays. Briefly, a silicone culture insert (Ibidi $\mathrm{GmbH}$; Martinsried, Germany) was used to generate the wound. Fadu cells were examined over 48 h under a Leica DMi8 microscope.

Cell invasion of Fadu cells treated with control siRNA or UVRAG siRNA was performed in BD Biocoat Matrigel Invasion Chambers ( $8 \mathrm{~mm}$ pore size, 24-well plate; $\mathrm{BD}$ Biosciences). Images were taken by a Leica DMi8 microscope.

\section{Cell Cycle Analysis}

Fadu cells treated with control siRNA or UVRAG siRNA were collected and incubated with $70 \%$ ethanol overnight. Propidium iodide (PI) supplemented with RNase (Becton Dickinson, San Diego, CA) was used to stain Fadu cells. Then cells were analyzed with a C6 flow cytometer (BD Biosciences; San Jose, CA, USA).

\section{Autophagic Flux Measurement}

Autophagic flux was tested by the Autophagy Tandem Sensor RFP-GFP-LC3B Kit (Thermo Fisher Scientific). Briefly, Fadu cells transfected with the RFP-GFP-LC3B reagent were treated with control siRNA or UVRAG siRNA. Forty-eight-hour later, cells were fixed and observed under a Leica TCS SP5 confocal microscope.

\section{Immunofluorescence}

DNA damage was determined using immunofluorescence staining of $\gamma-\mathrm{H} 2 \mathrm{AX}$ foci. Fadu cells treated with negative control siRNA, UVRAG siRNA, 4 Gy irradiation, UVRAG siRNA combining 4 Gy irradiation were incubated with $\gamma$-H2AX antibody (Cell Signaling Technology; Danvers, MA, USA) and Alexa Fluor 594 conjugated goat anti-rabbit secondary antibody (Abcam; Cambridge, UK). Red dots were counted under a Leica TCS SP5 Confocal Laser Scanning Microscope. 


\section{Colony Formation Assay}

Fadu cells were plated in six-well plates. Then cells were divided into four treatment group: control siRNA, 4 Gy irradiation (IR), UVRAG siRNA (siUVRAG) or a combination of siUVRAG and irradiation. After 14 days, cells were fixed and stained with crystal violet.

\section{Lysosomal Membrane Stability}

Lysosomal membrane stability was tested by transfection of GFP-fused galectin 3 (Obio, Shanghai, China) construct with lipofectamine 2000 reagent (ThermoFisher Scientific). Then Fadu cells were transfected with control siRNA or UVRAG siRNA as described above. Cells were observed with a Leica SP5 Confocal Microscope.

\section{Lactate Dehydrogenase (LDH) Assay}

Cytotoxic effect of different treatment groups was tested with a Cytotoxicity Detection Kit (Roche Applied Science, Basel, Switzerland). Briefly, cell lysis was centrifuged and the supernatants were collected to measure total cellular LDH after related treatment. The amount of released LDH from each group was measured at $490 \mathrm{~nm}$ by microplate reader (PerkinElmer).

\section{Statistical Analysis}

The GraphPad Prism software program (Version 6.07; La Jolla, CA, USA) was used to evaluate the unpaired t-tests. Results are presented as the mean \pm SE. $P$-values $<0.05$ were considered statistically significant.
A

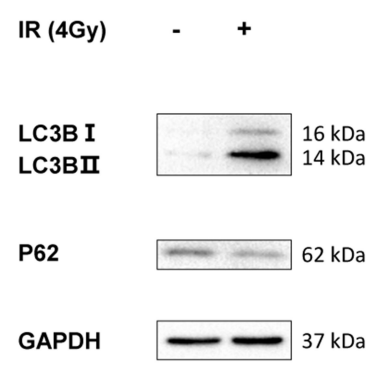

C

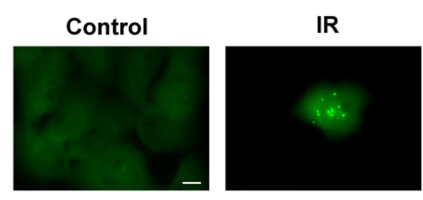

$\mathbf{F}$

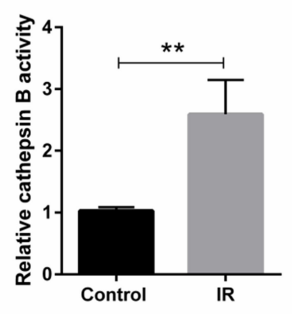

B
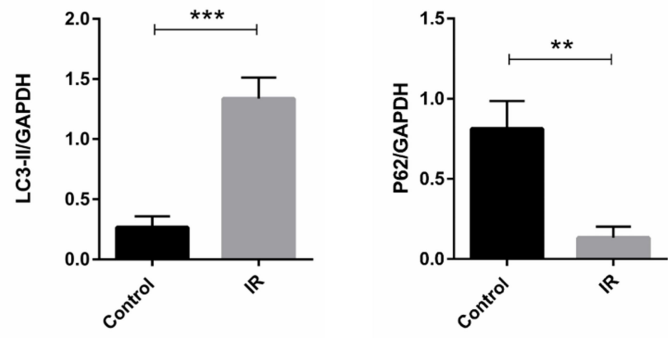

E

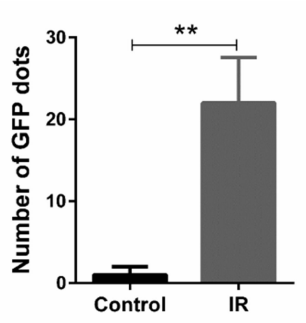

G

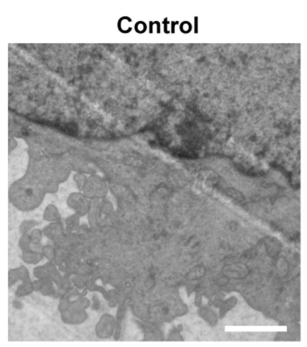

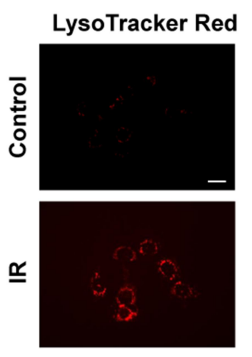
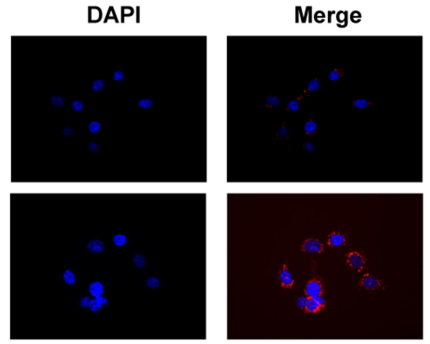

H
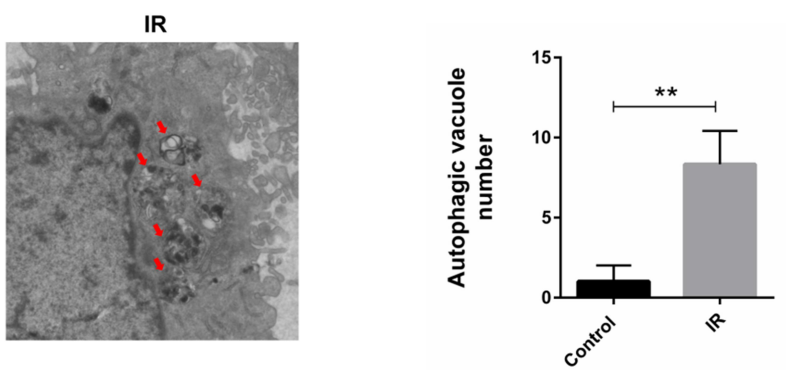

Figure I Irradiation increased autophagy in Fadu cells. (A) LC3B and p62 levels were examined by Western blot analysis in Fadu cells treated with or without 4 Gy irradiation. GAPDH was used as a loading control. (B) Densitometric analysis of the blots showed the ratios of LC3B-II and P62 to GAPDH. (C) GFP-LC3 transfection disclosed LC3 puncture in Fadu cells treated with or without 4 Gy irradiation. (D) Quantification of GFP-LC3 dots. (E) Representative images of Fadu cells stained with LysoTracker Red after treatment with or without 4 Gy irradiation. (F) Results of activity of cathepsin B in Fadu cells treated with or without 4 Gy irradiation. (G) Transmission electron microscopy image showing the ultrastructure of Fadu cells treated with or without 4 Gy irradiation. (H) Quantification of vesicles with double membrane. ${ }^{*} * P<0.01$; $* * * P<0.001$; scale bars in $(\mathbf{C})=10 \mu \mathrm{m}$, in $(\mathbf{E})=50 \mu \mathrm{m}$, in $(\mathbf{G})=1.2 \mu \mathrm{m}$. 


\section{Results}

\section{Irradiation Increased Autophagy in HSCC Cells}

Previous studies showed that irradiation increased autophagy in various tumor types. Here, we tried to find out whether irradiation increased autophagy in HSCC cells. We tested LC3B, a marker for the induction of autophagy. When autophagy occurs, LC3B-I is turned to LC3B-II. Western blot analysis illustrated that the protein levels of LC3B-II increased in irradiation treatment group compared with control group (Figure 1A and B). P62, an important protein which is degraded in autophagy, decreased in irradiation treatment group (Figure 1A and B). A GFP-LC3 expression construct was used to evaluate the formation of autophagosomes. Confocal microscopy illustrated increased green fluorescence puncta in irradiation treatment group (Figure 1C and D). LysoTracker Red, a lysosomotropic dye, was used to label lysosomes. Results showed that irradiation augmented the red fluorescence of LysoTracker Red
(Figure 1E), which indicated that lysosomal function was increased. We also found that the activity of cathepsin B, an important protease in the degradation of autophagosomes in lysosome, enhanced significantly after irradiation treatment (Figure 1F). Results of the transmission electron microscope indicated that many vesicles with double membrane accumulated (Figure $1 \mathrm{G}$ and $\mathrm{H}$ ). All these data suggested that irradiation increases autophagy in HSCC cells.

\section{Irradiation Upregulated UVRAG in HSCC}

As UVRAG is an important regulator of autophagy, we tried to study the expression of UVRAG in HSCC before and after irradiation treatment. Western blot analysis demonstrated that the UVRAG increased significantly after irradiation treatment (Figure 2A and B). We also collected three matched primary and recurrent HSCC tissues who had only received radiotherapy after first surgical resection. Results of immunohistochemistry showed that the expression of UVRAG increased significantly in
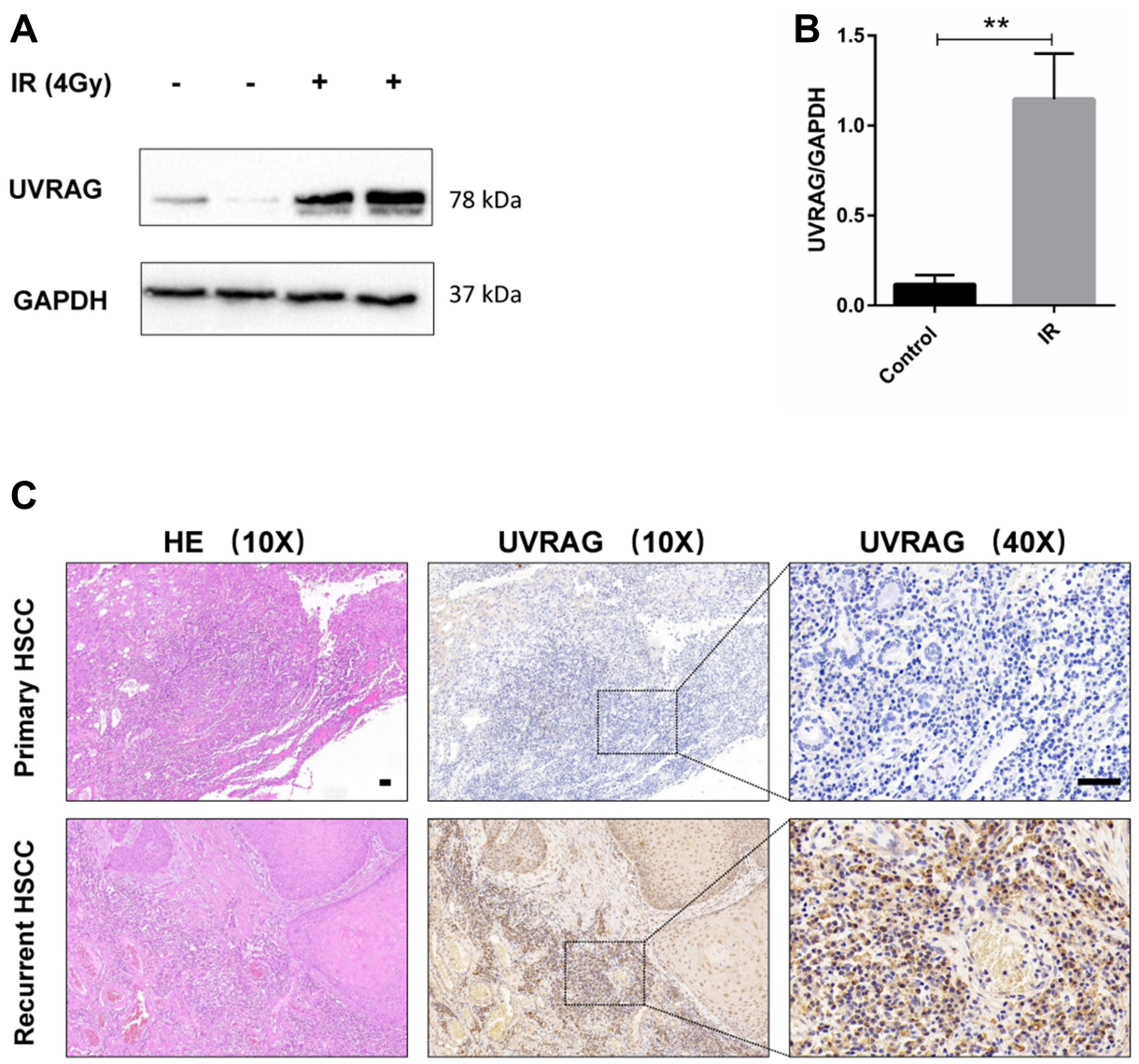

D

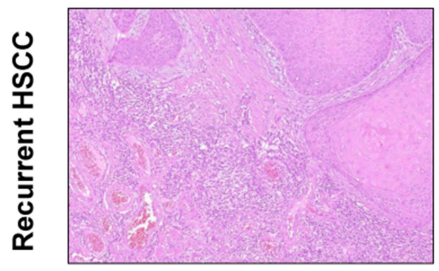

Figure 2 Irradiation upregulated UVRAG in HSCC. (A) Western blot analysis of UVRAG in Fadu cells treated with or without 4 Gy irradiation. GAPDH was used as a loading control. (B) Densitometric analysis of the blots showed the ratios of UVRAG to GAPDH. (C) Hematoxylin-eosin (HE) staining and immunohistochemistry of UVRAG in HSCC tumor tissues from primary and recurrent HSCC patients who have only received radiotherapy after first surgical resection. (D) Quantification of (C). $* * P<0.01$; Scale bars $=50 \mu \mathrm{m}$. 
recurrent $\mathrm{HSCC}$ tumor tissues compared with primary HSCC tumor tissues (Figure 2C and D). Altogether, these data demonstrated that the expression of UVRAG increased significantly after irradiation treatment.

\section{Knocking Down UVRAG Inhibited Cell Growth and Malignant Behaviors in HSCC Cells}

UVRAG was recognized as a tumor suppressor gene. But there are also studies showing that UVRAG contributes to tumorigenesis and chemo-resistance in colorectal cancer.${ }^{23,24}$ In our study, we tried to study the role of UVRAG in HSCC. Firstly, we knocked down UVRAG with a specific
siRNA and Western blot analysis verified the knocking down efficiency (Supplementary Figure 1). Results of CCK-8 assay showed that knocking down UVRAG decreased cell viability in HSCC cell line Fadu cells (Figure 3A). EdU assay showed that knocking down UVRAG inhibited cell proliferation of Fadu cells compared with control group (Figure 3B and C). Cell cycle analysis illustrated that knocking down UVRAG induced cell cycle arrest in G2-M phases (Figure 3D and E). Wound healing assay and Transwell assay demonstrated that inhibiting UVRAG decreased malignant behaviors like migration and invasion in Fadu cells (Figure 3F-I). These data indicated that UVRAG contributed to cell growth and malignant behaviors in Fadu cells.
A

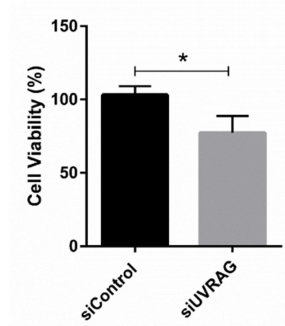

D
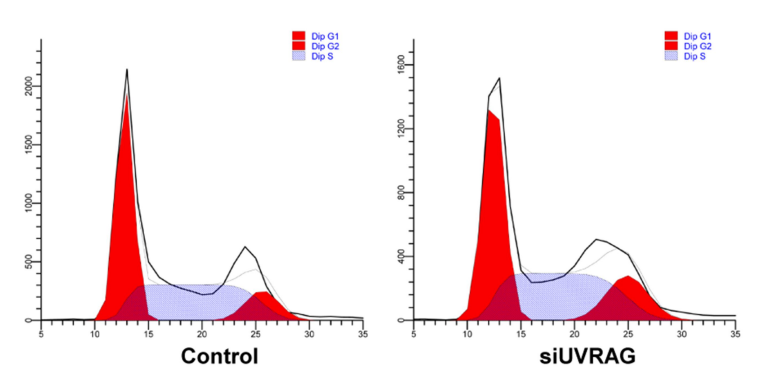

B

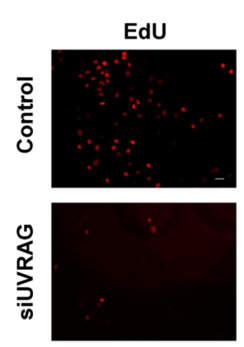

H

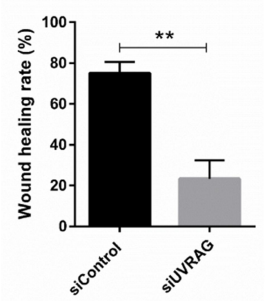

\section{G}

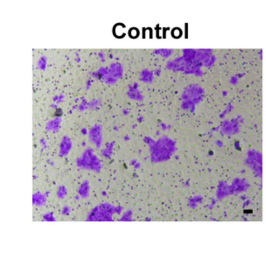

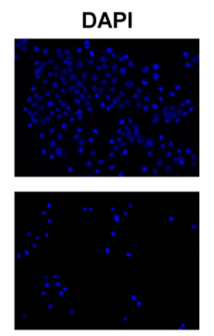

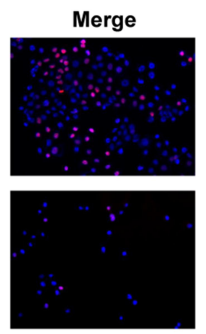

C

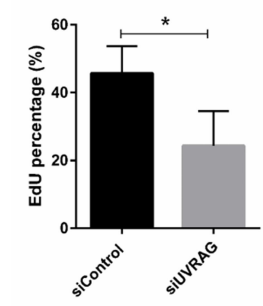

E

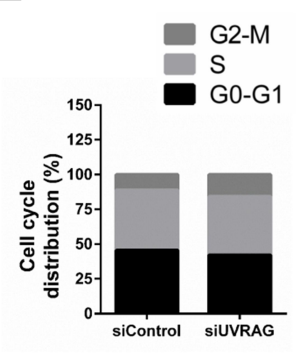

$\mathbf{F}$
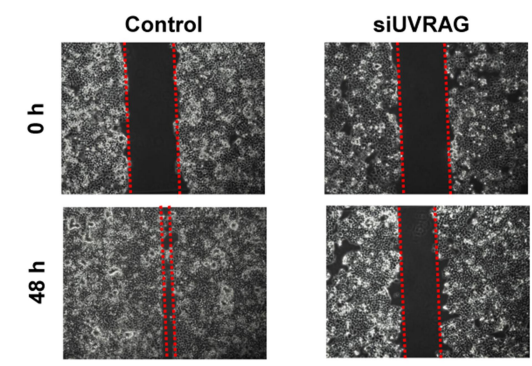

SIUVRAG

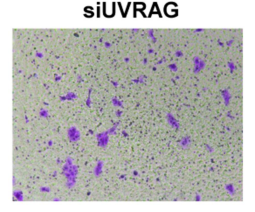

\section{I}

Figure 3 Knocking down UVRAG inhibited cell growth and malignant behaviors in Fadu cells. (A) Cell viability of Fadu cells treated with control or UVRAG siRNA. (B) EdU assay was used to test the proliferation in Fadu cells treated with control or UVRAG siRNA. (C) Quantification of EdU positive cells in (B). (D) Cell cycle analysis of Fadu cells treated with control or UVRAG siRNA. (E) Quantification of cells in GI, S, and G2/M. (F) Wound healing assay was used to study the migration of Fadu cells treated with control or UVRAG siRNA. (G) Quantification of (F). (H) Transwell assay was used to study the invasion of Fadu cells treated with control or UVRAG siRNA. (I) Quantification of $(\mathbf{H})$. ${ }^{*} P<0.05$; $* * P<0.01$; scale bars $=50 \mu \mathrm{m}$. 


\section{Inhibiting UVRAG Interfered Autophagy in Fadu Cells}

Considering the role of UVRAG in autophagy, we tried to study whether knocking down UVRAG inhibited autophagy in HSCC cells. We used rapamycin as an autophagy inducer to increase the basal level of autophagy in Fadu cells. Firstly, Western blot analysis showed that knocking down UVRAG decreased the protein levels of LC3B-II and increased the levels of P62 (Figure 4A and B). Then we used the Autophagy Tandem Sensor RFP-GFP-LC3B Kit (Thermo Fisher Scientific) to study autophagic flux. With an acid-sensitive green fluorescent protein (GFP) and an acid insensitive red fluorescent protein (RFP), the green fluorescence will disappear in the autolysosomes (with an acidic $\mathrm{pH}$ ), leaving only red fluorescence. Results showed that rapamycin increased red fluorescence dots significantly (Figure 4C and D). But in UVRAG knockdown group, rapamycin did not increase the number of green or red fluorescence dots (Figure 4C and D). All these data indicated that knocking down UVRAG interfered autophagy in Fadu cells.

\section{Knocking Down UVRAG Increased Radiosensitivity in Fadu Cells}

There are many DNA damage types, among which double strand breaks (DSBs) are the most lethal. Immunofluorescence of $\gamma$-H2AX, a marker of DSBs, is used to study whether knocking down UVRAG sensitized Fadu cells to irradiation treatment. Results showed that a combination of knocking down UVRAG and irradiation obviously increased red dots of $\gamma$-H2AX compared with cells treated with either siUVRAG or irradiation alone (Figure $5 \mathrm{~A}$ and $\mathrm{B}$ ). To further test the cytotoxic effect of irradiation in different treatment group, colony formation assay was carried out. Results showed that in combined treatment group, colony formation was inhibited the most (Figure 5C and D). Altogether, these data revealed that inhibiting UVRAG induced radiosensitization of Fadu cells.
A

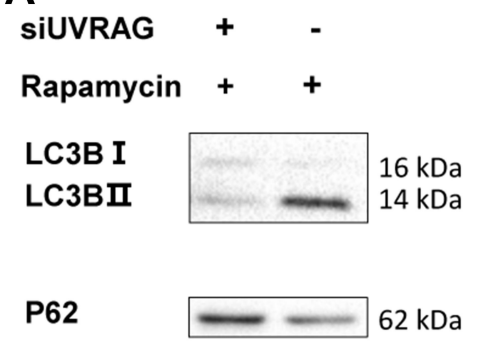

GAPDH

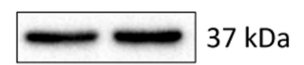

B

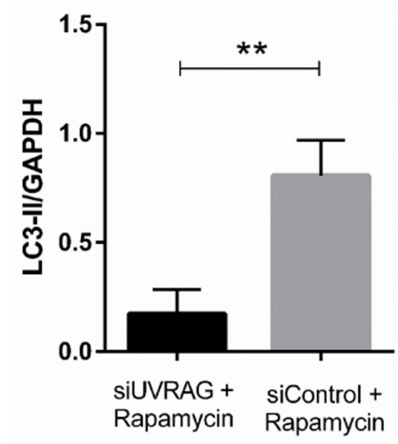

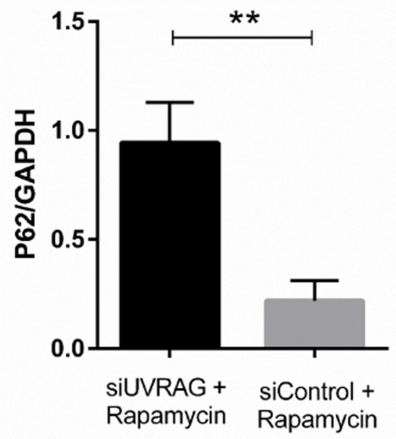

C

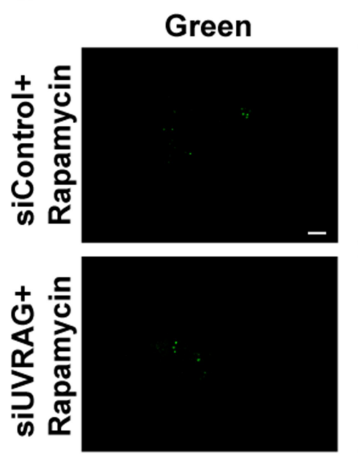

Red

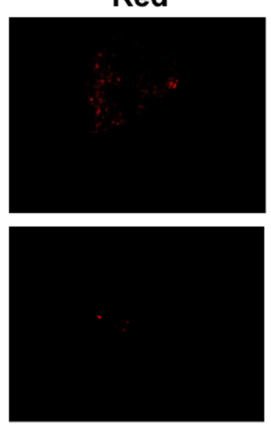

Merge

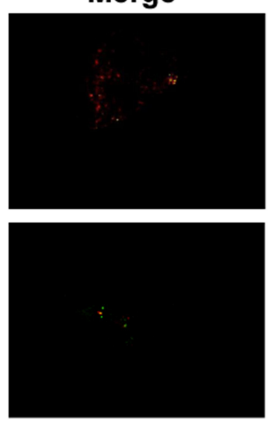

D

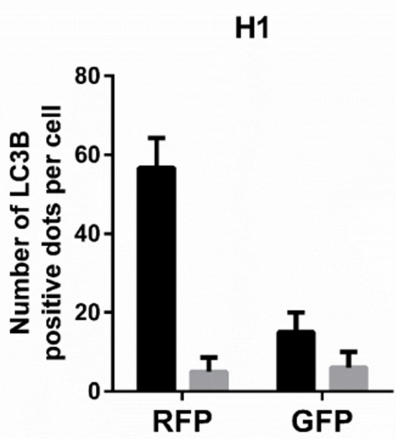

siControl +Rapamycin siUVRAG+Rapamycin

Figure 4 Inhibiting UVRAG interfered autophagy in Fadu cells. (A) LC3B and p62 levels were examined by Western blot analysis in Fadu cells treated with control or UVRAG siRNA. GAPDH was used as a loading control. Rapamycin was used to increase the basal level of autophagy. (B) Densitometric analysis of the blots showed the ratios of LC3B-II and P62 to GAPDH. (C) The Autophagy Tandem Sensor RFP-GFP-LC3B Kit was used to study autophagic flux in Fadu cells treated with control or UVRAG siRNA. (D) Quantification of red and green dots in (C). ${ }^{*} P P<0.01$; size bars $=10 \mu \mathrm{m}$. 
A
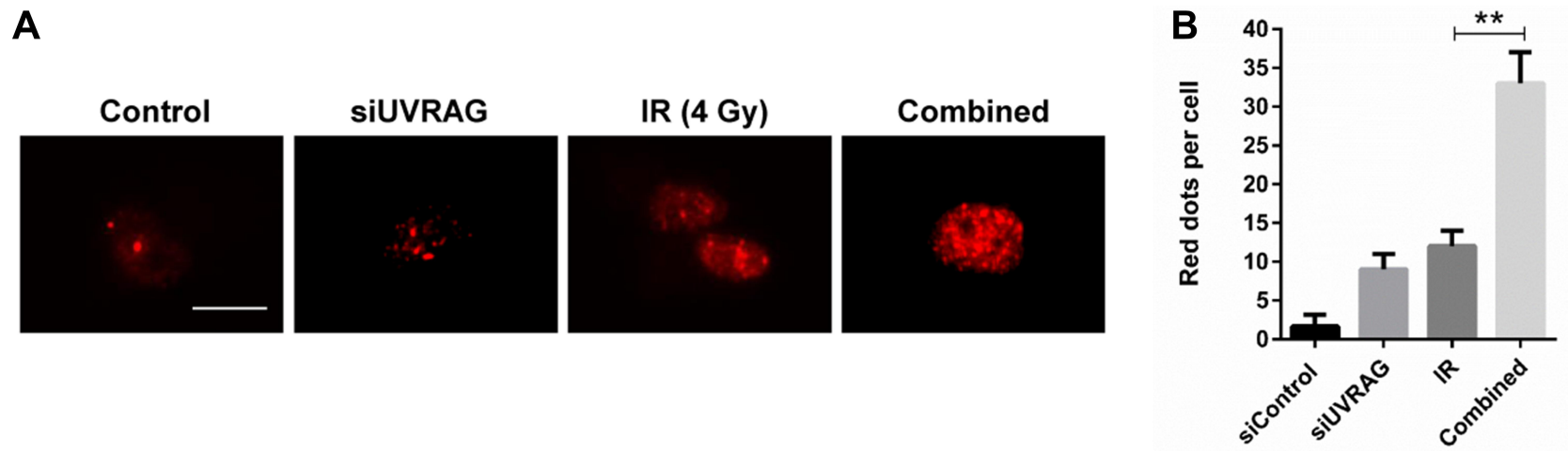

C
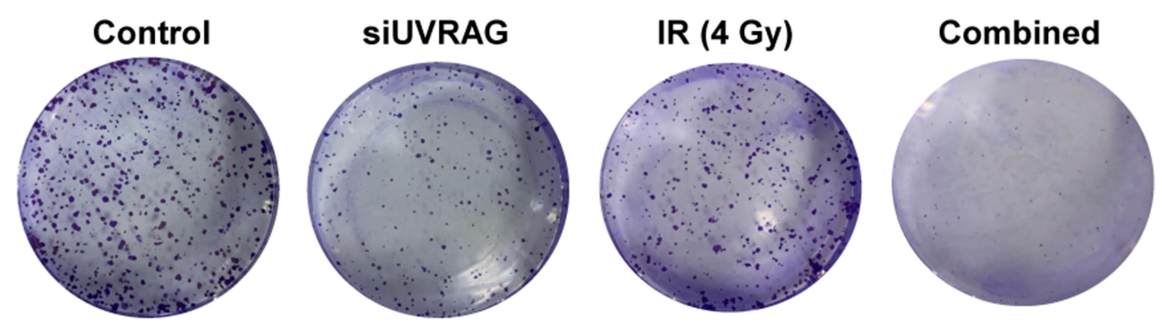

D

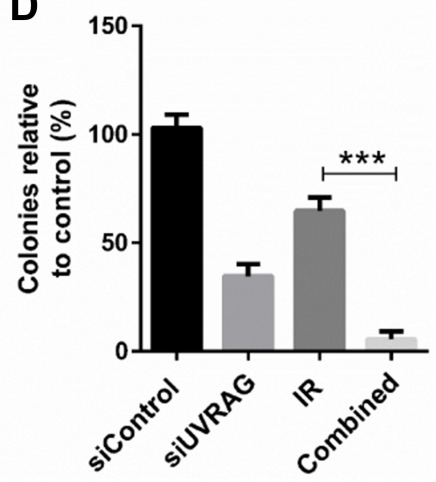

Figure 5 Knocking down UVRAG increased radiosensitivity in Fadu cells. (A) Immunofluorescence staining of $\gamma$ - $\mathrm{H} 2 \mathrm{AX}$ (red fluorescence) to determine the double strand breaks (DSBs) caused by control siRNA, 4 Gy irradiation (IR), UVRAG siRNA (siUVRAG) or a combination of siUVRAG and irradiation. (B) Quantification of red puncta associated with $\gamma-\mathrm{H} 2 \mathrm{AX}$ in (A). (C) Colony formation of Fadu cells treated with control siRNA, 4 Gy irradiation (IR), UVRAG siRNA (siUVRAG) or a combination of siUVRAG and irradiation. (D) Quantification of (C). ${ }^{* *} P<0.01$; ***P $<0.001$; size bars $=10 \mu \mathrm{m}$.

\section{Suppressing UVRAG Induced Lysosomal Membrane Permeabilization, Which Contributed to Radiosensitization in Fadu Cells}

Previous study found that UVRAG is involved in regulating the function and morphology of lysosomes and knocking down UVRAG led to enlarged lysosomes. ${ }^{19}$ Lysosomal membrane permeabilization (LMP) is characterized for enlarged lysosomes. Here we tried to study whether knocking down UVRAG induced LMP. With GFP-fused galectin 3 transient transfection, we found that inhibiting UVRAG caused green fluorescent dots, while GFP-fused galectin 3 was distributed evenly in negative control group (Figure 6A).

LMP is recognized as another factor contributing to radiosensitization. ${ }^{7}$ In the process of LMP, lysosomal proteases are released into the cytoplasm, which activating a series of programmed cell death pathways. Cathepsin $\mathrm{B}$, a cysteine protease, plays an important role in LMP. Then we used CA$074 \mathrm{Me}$, a specific inhibitor of cathepsin B, to test whether the radiosensitive effect caused by knocking down UVRAG is
LMP-dependent. Results of LDH assay showed that a combination of CA- $074 \mathrm{Me}$, irradiation and siUVRAG led to less cell death compared to irradiation following knocking down UVRAG treatment (Figure 6B). All these data suggested that suppressing UVRAG induced lysosomal membrane permeabilization, which contributed to radiosensitization in Fadu cells.

\section{Discussion}

Hypopharyngeal squamous cell carcinoma (HSCC) is one of the most malignant head and neck cancers. ${ }^{25}$ Although there is a comprehensive treatment including surgical excision, chemotherapy and radiotherapy, the prognosis of patients with HSCC remains poor. ${ }^{26}$ Radioresistance is one of the most important factors leading to treatment failure of $\mathrm{HSCC}^{27}$ As a result, finding out the underlying mechanisms leading to radioresistance will help develop new therapy to improve prognosis of patients with HSCC. Until now, many mechanisms have been disclosed, like DNA damage repair, apoptotic resistance, lysosomal membrane permeabilization, ${ }^{7}$ cell cycle arrest and autophagy. ${ }^{28}$ Autophagy is a lysosome-dependent 
A
siControl

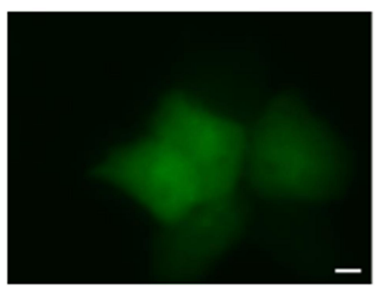

SIUVRAG

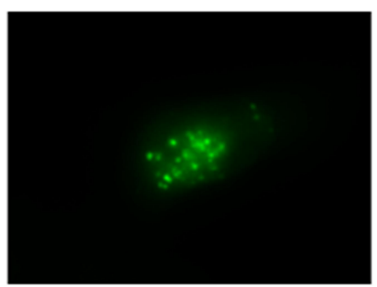

B

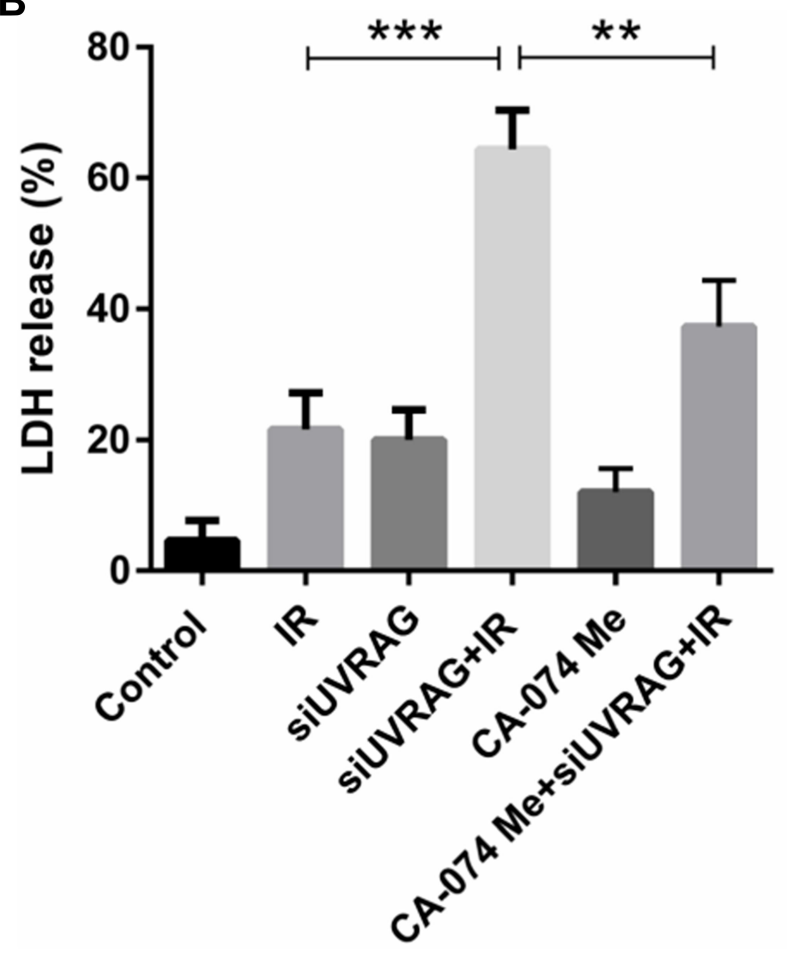

Figure 6 Suppressing UVRAG induced lysosomal membrane permeabilization, which contributed to radiosensitization in Fadu cells (A) GFP-fused galectin 3 construct was used to study LMP in Fadu cells treated with control siRNA (siControl) or UVRAG siRNA (siUVRAG). (B) Fadu cells were divided into six treatment group: control, 4 Gy irradiation (IR), UVRAG siRNA (siUVRAG), siUVRAG + 4 Gy IR, $5 \mu$ M CA-074 Me, siUVRAG + 4 Gy IR + $5 \mu$ M CA-074 Me. LDH assay was used to study the cytotoxic effect of different groups. **P $<0.01$; *** $P<0.001$; size bars $=10 \mu \mathrm{m}$.

process in which unfunctional and damaged organelles are degraded and recycled for energy and further biogenesis, ${ }^{5}$ and it is recognized as one of self-protection against internal and external damages. Many studies found that after radiotherapy, autophagy was enhanced and inhibiting autophagy increased apoptosis induced by irradiation. ${ }^{5,12,14}$ What's more, inhibiting autophagy also impaired DNA damage repair and increased irradiationinduced DNA damage. ${ }^{5}$ In our study, we found that irradiation increased the level of autophagy in HSCC cell line Fadu cells.
Ultraviolet irradiation resistance-associated gene (UVRAG) is well known for its role in autophagy regulation. UVRAG has two most important roles in the process of autophagy, promoting autophagosome formation and autophagosome maturation. ${ }^{17}$ However, more and more studies show that UVRAG has many other functions except for autophagy, including cell death regulation, endocytosis and immunity-related GTPase transport. ${ }^{18}$ UVRAG is also involved in the regulation of lysosomal function and morphology. ${ }^{19}$ UVRAG is originally recognized as a tumor suppressor gene. ${ }^{29}$ UVRAG gene is located on the $11 \mathrm{q} 13$ chromosome band, a relevant area with oncogenic implications in many cancers, mainly due to its frequent amplification and the genes it houses. ${ }^{30}$ However, accumulating evidence indicates that UVRAG suppresses apoptosis, and knocking down UVRAG increases apoptotic rate in tumor cells. ${ }^{31}$ Besides, UVRAG is also involved in DNA damage repair and maintaining chromosomal stability. ${ }^{20}$ Chang et al found that silencing UVRAG reversed the radioresistant phenotypes and led to increased apoptosis, impaired DNA repair. $^{22}$ In our study, irradiation increased protein levels of UVRAG not only in Fadu cells, but also in tumor tissues from recurrent HSCC patients who had only received radiotherapy after surgical resection. This is the first study showing that UVRAG is upregulated in tumor tissues from irradiated recurrent HSCC patients. We found that knocking down UVRAG with a specific siRNA inhibited cell growth, caused cell cycle arrest and inhibited malignant behaviors in HSCC cell line Fadu cells. Our study showed that inhibiting UVRAG led to lysosomal membrane permeabilization (LMP), which contributed to the radiosensitizing effect of knocking down UVRAG in Fadu cells. Our study supports the role of lysosome in the radioresistance of cancer. ${ }^{6}$

Many factors trigger LMP, among which ROS is the most studied. ${ }^{7}$ ROS and autophagy are two important factors involved in cellular stress response. ${ }^{32}$ Previously, many studies reported that ROS was a key inducer of autophagy. $^{33,34}$ But recently, more and more studies showed that inhibition of autophagy could also upregulate the level of ROS. ${ }^{35,36}$ Autophagy defection induced by UVRAG inhibition may cause upregulation of ROS, which leads to LMP. But further studies are warranted.

We have to recognize that limitations existed in our study. All these experiments were only repeated in one cell line, decreasing the internal validity of results. More studies are warranted in other head and neck tumor cell lines. Experiments were not blinded. Sample size was too small 
for immunohistochemical analysis. More matched tumor tissues are needed in the future.

\section{Conclusion}

In this study, we found that irradiation increased autophagy in HSCC cells. Protein levels of UVRAG were also increased in irradiated Fadu cells and tumor tissues from recurrent HSCC patients who had only received radiotherapy. Our study demonstrates that UVRAG contributes to tumor development in HSCC cells, and knocking down UVRAG induces LMP, which contributes to the radiosensitive effect of knocking down UVRAG in Fadu cells. Altogether, our study revealed UVRAG as a promising antitumor target in HSCC.

\section{Acknowledgments}

This work was supported by the Medical Science Technology Development Program of Shandong Province (2018WS103).

\section{Disclosure}

The authors report no conflicts of interest in this work.

\section{References}

1. Li Y, Liu C, Wang Z, Hu G. Expression of protocadherin8: function as a tumor suppressor in hypopharyngeal carcinoma. Cancer Biomark. 2018;22(3):495-502. doi:10.3233/CBM-171137

2. Tsou YA, Chang WD, Lu JJ, et al. The effect of metformin use on hypopharyngeal squamous cell carcinoma in diabetes mellitus patients. BMC Cancer. 2019;19(1):862. doi:10.1186/s12885-019-6083-5

3. Bray F, Ferlay J, Soerjomataram I, Siegel RL, Torre LA, Jemal A. Global cancer statistics 2018: GLOBOCAN estimates of incidence and mortality worldwide for 36 cancers in 185 countries. CA Cancer $J$ Clin. 2018;68(6):394-424. doi:10.3322/caac.21492

4. Wang H, Wang Z, Li Y, Lu T, Hu G. Silencing snail reverses epithelial-mesenchymal transition and increases radiosensitivity in hypopharyngeal carcinoma. Onco Targets Ther. 2020;13:497-511. doi:10.2147/OTT.S237410

5. Zhang X, Xu R, Zhang C, et al. Trifluoperazine, a novel autophagy inhibitor, increases radiosensitivity in glioblastoma by impairing homologous recombination. J Exp Clin Cancer Res. 2017;36(1):118. doi:10.1186/s13046-017-0588-z

6. Zhang X, Wang J, Li X, Wang D. Lysosomes contribute to radioresistance in cancer. Cancer Lett. 2018;439:39-46. doi:10.1016/j. canlet.2018.08.029

7. Zhou W, Guo Y, Zhang X, Jiang Z. Lys05 induces lysosomal membrane permeabilization and increases radiosensitivity in glioblastoma. J Cell Biochem. 2020;121(2):2027-2037. doi:10.1002/jcb.29437

8. Wang XH, Zhang ZH, Cai XL, et al. Lipopolysaccharide induces autophagy by targeting the AMPK-mTOR pathway in human nasal epithelial cells. Biomed Pharmacother. 2017;96:899-904. doi:10.1016/ j.biopha.2017.12.011

9. Li L, Wang Y, Jiao L, et al. Protective autophagy decreases osimertinib cytotoxicity through regulation of stem cell-like properties in lung cancer. Cancer Lett. 2019;452:191-202. doi:10.1016/j.canlet.2019. 03.027
10. Meng Q, Xu J, Liang C, et al. GPx1 is involved in the induction of protective autophagy in pancreatic cancer cells in response to glucose deprivation. Cell Death Dis. 2018;9(12):1187. doi:10.1038/s41419018-1244-z

11. Lin JF, Lin YC, Tsai TF, Chen HE, Chou KY, Hwang TI. Cisplatin induces protective autophagy through activation of BECN1 in human bladder cancer cells. Drug Des Devel Ther. 2017;11:1517-1533. doi:10.2147/DDDT.S126464

12. Pietrocola F, Pol J, Vacchelli E, et al. Autophagy induction for the treatment of cancer. Autophagy. 2016;12(10):1962-1964. doi:10.1080/15548627.2016.1214778

13. Wang F, Xia X, Yang C, et al. SMAD4 gene mutation renders pancreatic cancer resistance to radiotherapy through promotion of autophagy. Clin Cancer Res. 2018;24(13):3176-3185. doi:10.1158/ 1078-0432.CCR-17-3435

14. Lee TG, Kim SY, Kim HR, Kim H, Kim CH. Radiation induces autophagy via histone $\mathrm{H} 4$ lysine 20 trimethylation in non-small cell lung cancer cells. Anticancer Res. 2020;40(5):2537-2548. doi:10.21873/anticanres.14224

15. Feng X, Jia Y, Zhang Y, et al. Ubiquitination of UVRAG by SMURF1 promotes autophagosome maturation and inhibits hepatocellular carcinoma growth. Autophagy. 2019;15(7):1130-1149. doi:10.1080/15548627.2019.1570063

16. Quach C, Song Y, Guo H, et al. A truncating mutation in the autophagy gene UVRAG drives inflammation and tumorigenesis in mice. Nat Commun. 2019;10(1):5681. doi:10.1038/s41467-019-13475-w

17. Song Y, Quach C, Liang C. UVRAG in autophagy, inflammation, and cancer. Autophagy. 2020;16(2):387-388. doi:10.1080/15548627. 2019.1709768

18. Yin X, Cao L, Kang R, et al. UV irradiation resistance-associated gene suppresses apoptosis by interfering with BAX activation. EMBO Rep. 2011;12(7):727-734. doi:10.1038/embor.2011.79

19. Munson MJ, Allen GF, Toth R, Campbell DG, Lucocq JM, Ganley IG. mTOR activates the VPS34-UVRAG complex to regulate autolysosomal tubulation and cell survival. EMBO J. 2015;34(17):22722290. doi:10.15252/embj.201590992

20. Zhao Z, Oh S, Li D, et al. A dual role for UVRAG in maintaining chromosomal stability independent of autophagy. Dev Cell. 2012;22 (5):1001-1016. doi:10.1016/j.devcel.2011.12.027

21. Park JM, Tougeron D, Huang S, Okamoto K, Sinicrope FA. Beclin 1 and UVRAG confer protection from radiation-induced DNA damage and maintain centrosome stability in colorectal cancer cells. PLoS One. 2014;9(6):e100819. doi:10.1371/journal.pone.0100819

22. Chang VH, Tsai YC, Tsai YL, et al. Krupple-like factor 10 regulates radio-sensitivity of pancreatic cancer via UV radiation resistanceassociated gene. Radiother Oncol. 2017;122(3):476-484. doi:10.1016/j.radonc.2017.01.001

23. Huangfu L, Liang $\mathrm{H}$, Wang $\mathrm{G}$, et al. miR-183 regulates autophagy and apoptosis in colorectal cancer through targeting of UVRAG. Oncotarget. 2016;7(4):4735-4745. doi:10.18632/oncotarget.6732

24. Jo YK, Park NY, Shin JH, et al. Up-regulation of UVRAG by HDAC1 inhibition attenuates 5FU-induced cell death in HCT116 colorectal cancer cells. Anticancer Res. 2018;38(1):271-277.

25. Powell SF, Gold KA, Gitau MM, et al. Safety and efficacy of pembrolizumab with chemoradiotherapy in locally advanced head and neck squamous cell carcinoma: a Phase IB Study. $J$ Clin Oncol. 2020;38(21)JCO1903156.

26. Janoray G, Pointreau Y, Alfonsi M, et al. Induction chemotherapy followed by cisplatin or cetuximab concomitant to radiotherapy for laryngeal/hypopharyngeal cancer: long-term results of the TREMPLIN randomised GORTEC trial. Eur $J$ Cancer. 2020;133:86-93. doi:10.1016/j.ejca.2020.04.009

27. Liu C, Liao K, Gross N, et al. Homologous recombination enhances radioresistance in hypopharyngeal cancer cell line by targeting DNA damage response. Oral Oncol. 2020;100:104469. doi:10.1016/j. oraloncology.2019.104469 
28. Zhan Y, Fan S. Multiple mechanisms involving in radioresistance of nasopharyngeal carcinoma. J Cancer. 2020;11(14):4193-4204.

29. Yang Y, He S, Wang Q, et al. Autophagic UVRAG promotes UVinduced photolesion repair by activation of the CRL4(DDB2) E3 ligase. Mol Cell. 2016;62(4):507-519. doi:10.1016/j.molcel.2016.04.014

30. Ramos-Garcia P, Ruiz-Avila I, Gil-Montoya JA, et al. Relevance of chromosomal band 11q13 in oral carcinogenesis: an update of current knowledge. Oral Oncol. 2017;72:7-16. doi:10.1016/j.oraloncology. 2017.04.016

31. Yin X, Cao L, Peng Y, et al. A critical role for UVRAG in apoptosis. Autophagy. 2011;7(10):1242-1244. doi:10.4161/auto.7.10.16507

32. Dhar SK, Bakthavatchalu V, Dhar B, et al. DNA polymerase gamma (polgamma) deficiency triggers a selective mTORC2 prosurvival autophagy response via mitochondria-mediated ROS signaling. Oncogene. 2018;37(48):6225-6242.
33. Huang Y, Li Y, Lou A, et al. Alamandine attenuates hepatic fibrosis by regulating autophagy induced by NOX4-dependent ROS. Clin Sci (Lond). 2020;134(7):853-869. doi:10.1042/CS20191235

34. Sharma M, Pandey R, Saluja D. ROS is the major player in regulating altered autophagy and lifespan in sin-3 mutants of C. elegans. Autophagy. 2018;14(7):1239-1255. doi:10.1080/15548627.2018.1474312

35. Datta S, Choudhury D, Das A, et al. Autophagy inhibition with chloroquine reverts paclitaxel resistance and attenuates metastatic potential in human nonsmall lung adenocarcinoma A549 cells via ROS mediated modulation of beta-catenin pathway. Apoptosis. 2019;24(5-6):414-433. doi:10.1007/s10495-019-01526-y

36. Li Q, Yin Y, Zheng Y, Chen F, Jin P. Inhibition of autophagy promoted high glucose/ROS-mediated apoptosis in ADSCs. Stem Cell Res Ther. 2018;9(1):289. doi:10.1186/s13287-018-1029-4

\section{Publish your work in this journal}

OncoTargets and Therapy is an international, peer-reviewed, open access journal focusing on the pathological basis of all cancers, potential targets for therapy and treatment protocols employed to improve the management of cancer patients. The journal also focuses on the impact of management programs and new therapeutic agents and protocols on patient perspectives such as quality of life, adherence and satisfaction. The manuscript management system is completely online and includes a very quick and fair peer-review system, which is all easy to use. Visit http://www.dovepress.com/ testimonials.php to read real quotes from published authors. 